\title{
ANÁLISIS PSICOSOCIAL DE LAS BARRERAS QUE DIFICULTAN LA DENUNCIA: EL CASO DE LOS FEMICIDIOS ÍNTIMOS EN ESPAÑA
}

\section{Resumen}

La evidencia disponible muestra que, en España, sólo una cuarta parte de las mujeres víctimas de femicidio íntimo habían denunciado previamente la violencia padecida (25,8\% desde 2005). El objetivo de este trabajo es contribuir a identificar y comprender las barreras y dificultades para denunciar que encontraron estas mujeres. Se realizó un estudio cualitativo, entrevistando a 34 profesionales y 5 personas significativas relacionadas con los 23 casos ocurridos entre 2004 y 2014 en Baleares. Las principales razones para no denunciar fueron la falta de percepción de peligro y las limitaciones de los recursos comunitarios y/o jurídicos para atender a estas mujeres. Este estudio también mostró las consecuencias de estos femicidios para el entorno cercano de las víctimas.

Palabras clave: violencia contra las mujeres en la pareja, femicidio íntimo, denuncias, factores psicosociales.

\section{Resumo \\ Análise psicossocial das barreiras que dificultam a denúncia: o caso do femicídio íntimo em Espanha}

As evidências mostram que, em Espanha, apenas uma quarta parte das mulheres vítimas de femicídio íntimo denunciou previamente a violência sofrida $(25,8 \%$ desde 2005). O objetivo deste trabalho é ajudar a identificar e compreender as barreiras e dificuldades de denúncia reconhecidas por estas mulheres. Para isso, realizou-se um estudo qualitativo, tendo-se entrevistado 34 profissionais e 5 pessoas significativas das vítimas sobre 23 casos ocorridos entre 2004 e 2014 nas ilhas Baleares. Os resultados mostram que as principais razões para não denunciar a violência são a falta de perceção de risco e as limitações da comunidade e dos recursos legais. Este estudo também mostrou as consequências dos femicídios para o ambiente social das vítimas.

Palavras-chave: violência na intimidade contra as mulheres, femicídio íntimo, queixas, fatores psicossociais.

\footnotetext{
Grupo de Investigación de Estudios de Género, Universidad de las Islas Baleares, 07122 Palma, Islas Baleares, España.

Correo electrónico: victoria.ferrer@uib.es; esperanza.bosch@uib.es

Dirección postal de las autoras: Facultad de Psicología. Campus de la UIB. Ctra. Valldemossa km. 7'5. 07122 Palma (Baleares) (España).
}

Agradecimientos: Este trabajo se realizó en el marco del proyecto ARIADNA, subvencionado por el Instituto Balear de la Mujer y los EEAGrants. Las autoras agradecen la colaboración en la recogida y codificación de la información a Enrique Urbano, María Vicens, Raquel Herrezuelo y Elena Nájera. 


\begin{abstract}
Analysis of psychosocial barriers that hinder the complaint: the case of intimate femicides in Spain

Evidence shows that, in Spain, only a quarter of women victim of intimate femicide had previously reported the violence suffered (25,8\% from 2005). The aim of this research is to help identify and understand the barriers and difficulties in reporting faced by these women. For this, a qualitative study was conducted, interviewing 34 professionals and 5 significant others related to 23 cases that occurred between 2004 and 2014 in the Balearic Islands. The results show that the main reasons for not reporting the violence were the lack of perception of risk, and the limitations of community and legal resources. This study also shows the consequences of these femicides for the social environment of the victims.
\end{abstract}

Keywords: intimate partner violence against women, intimate femicide, complaints, psychosocial factors.

\title{
Introducción
}

La violencia contra las mujeres en la pareja (denominada violencia de género en el ordenamiento jurídico español) tiene consecuencias sobre la salud física, mental y social de quienes la padecen, y también consecuencias fatales, incluyendo homicidios, suicidios, mortalidad materna y muertes relacionadas con el VIH (García-Moreno et al. 2013). Este trabajo se centra en los femicidios íntimos (Manjoo 2012), esto es, los asesinatos de mujeres a manos de sus parejas o exparejas. El término femicidio fue introducido por Diana Russell en 1976 para referirse al asesinato de mujeres y niñas por motivos de género (Russell 2013), y como tal es reconocido (ONU 2012) y empleado actualmente en análisis sociológicos y criminológicos (Spinelli 2013).

Concretamente, en este trabajo se analiza, desde un punto de vista psicosocial, los factores que influyen en que mujeres víctimas de femicidio íntimo hubieran (o no) denunciado previamente la violencia vivida. Ampliar el conocimiento sobre esta cuestión permitiría aportar claves útiles para la prevención terciaria y para mejorar la protección a quienes se hallan en esta situación (Amnistía Internacional 2012).

La cuestión de las denuncias en casos de violencia de género se ha abordado desde diferentes puntos de vista y ha generado algunas controversias sobre si debe o no ser un paso necesario para acceder a mecanismos de protección y ayuda (Castillejo 2009; Naredo 2009), si debe o no existir una respuesta estandarizada a todos los casos (Castillejo 2011; Casado 2012; Laliga y Bonilla 2015), o si poner énfasis en la necesidad de denunciar puede suponer culpabilizar a las mujeres por la violencia padecida (Castillejo 2011; Naredo 2009; Amnistía Internacional 2012; Laliga y Bonilla 2015). Por ello, se considera necesario puntualizar que en este trabajo se adopta un punto de vista psicosocial, se entiende la denuncia como demanda de ayuda con efecto terapéutico (OPS 2013), y se parte de una 
consideración activa de las mujeres que padecen violencia de género (Cala y García 2014).

\section{Las denuncias por violencia de género en España}

A pesar de los avances en su reconocimiento y progresiva visibilización, puede seguir afirmándose que la violencia de género es un problema oculto cuyas cifras reales son difíciles de conocer (Harway 2002; Gracia 2009; Palermo, Bleck y Peterman 2014; Sierra et al. 2014).

En este contexto, las denuncias ofrecen información sobre violencia declarada o reported violence (EIGE 2014) y, en España, fueron la primera fuente de información sistemática disponible a partir de 1983. Las cifras han variado considerablemente desde entonces, oscilando entre las 11516 denuncias recogidas en 1983 y las 129123 en 2015. ${ }^{1}$ Algunos autores consideran que en España existe una tendencia general creciente a denunciar el maltrato (Meil 2012). En relación con esto, cabe recordar que una mayor tasa de denuncias puede interpretarse como señal de mayor confianza de las mujeres en los Estados para combatir la violencia, mayor ejercicio del derecho a la reparación o menor tolerancia de la violencia, pero también de mejoras en los procedimientos de registro (García-Moreno y Jansen 2009). Sin embargo, hasta la fecha no hay estudios empíricos que demuestren la existencia de una relación causal entre estos cambios y el aumento de denuncias (Blay 2013). Además, esta tendencia no es constante y los datos disponibles no son estrictamente comparables, pues, al margen del posible aumento del número de casos, se han introducido importantes modificaciones legislativas y en los criterios de recogida (Ferrer, Bosch y Riera 2006; Novo y Seijo 2009; Blay 2013).

En cualquier caso, en todo este proceso, la Ley Orgánica 1/2004, de Medidas de Protección Integral contra la Violencia de Género, supuso un punto de inflexión importante (Pontón et al. 2015) al delimitar el concepto de violencia de género e incorporar cambios legislativos, asistenciales y policiales, y también metodológicos para la recogida de información. Entre 2005, cuando entró en vigor esta ley, y 2015 se cursaron en España 1303294 denuncias por violencia de género (una media de 118481 denuncias al año, 325 denuncias al día). ${ }^{2}$ Según estas mismas fuentes, Baleares es una de las comunidades autónomas con mayor tasa de denuncias por violencia de género (i.e., 82,5 por cada 10000 mujeres en 2015).

\footnotetext{
1 Informes estadísticos periódicos disponibles en: <http:// estadisticasviolenciagenero.msssi. gob.es> y <http: / / www.poderjudicial.es/cgpj/es / Temas / Violencia-domestica-y-degenero / Actividad-del-Observatorio/Informes-de-violencia-domestica>.

2 Ver nota anterior.
} 


\section{¿Cuánta violencia de género se denuncia?}

$\mathrm{Al}$ no disponer de información sobre el número real de casos, resulta difícil determinar cuánta violencia llega a denunciarse (Ferrer, Bosch y Riera 2006). Comparar el número de denuncias presentadas y los resultados de encuestas sobre el tema permite realizar una estimación.

A nivel internacional (García-Moreno 2005; Akers y Kaukinen 2009; Palermo, Bleck y Peterman 2014) se estima que el número de denuncias interpuestas se correspondería, aproximadamente, a entre un 5\% y un $30 \%$ de los casos de violencia de género realmente existentes. Las más recientes encuestas que preguntan directamente por denuncias interpuestas (Ministerio de Sanidad, Servicios Sociales e Igualdad 2012; FRA 2014; DGVG 2015a) indican que en España se denunciaría, aproximadamente, un $25 \%$ de la violencia de género. En los femicidios íntimos ocurridos en España, se observan tasas de denuncia similares (Figura 1), con un promedio del 25,8\% desde 2005 (oscilando entre un $19,2 \%$, en 2012 , y un $31,9 \%$, en 2006).

\section{Figura 1}

Mujeres muertas a manos de sus parejas y exparejas en España

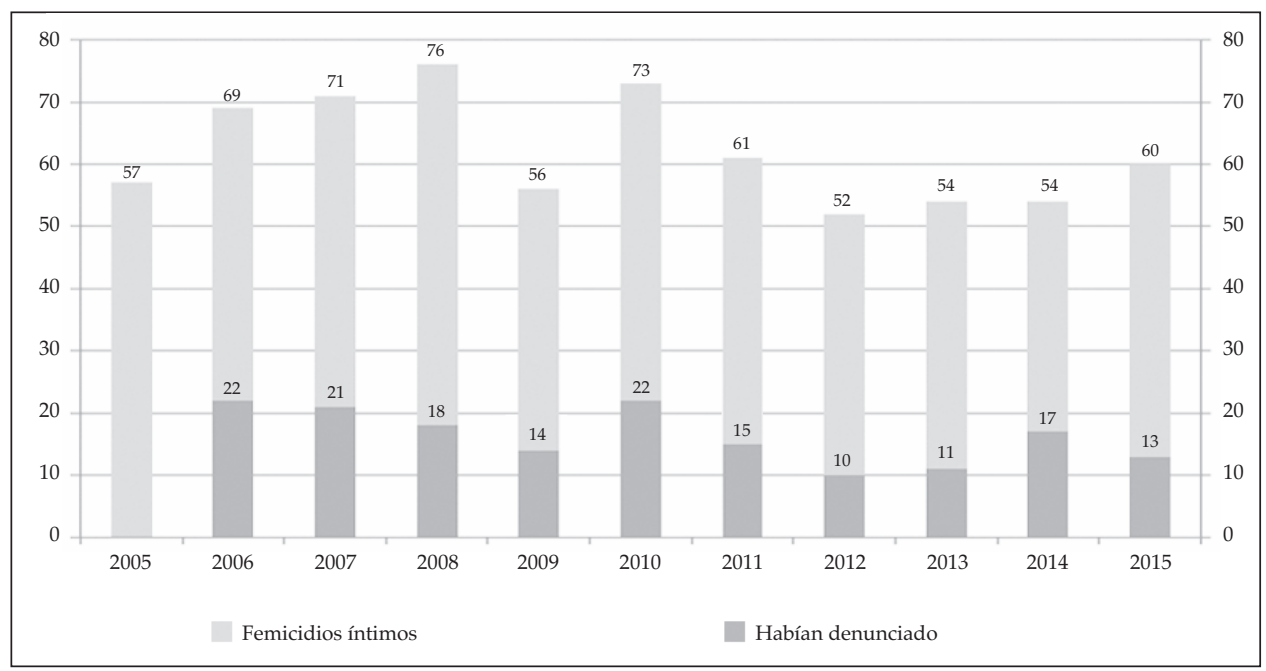

Nota: Para 2005 no se dispone del dato sobre denuncias.

Fuente: Elaboración propia a partir de datos del Portal Estadístico de la Delegación del Gobierno para la Violencia de Género (http://estadisticasviolenciagenero.msssi.gob.es).

En definitiva, en España sólo 1 de cada 4 mujeres denuncia la violencia que padece a manos de su pareja o expareja (tanto en general como en casos de femicidio íntimo), y, aunque esta proporción parece haber aumentado ligeramente, dicho incremento no es remarcable ni continuado. 


\section{Las barreras para denunciar la violencia de género}

La literatura previa sobre el tema (Felson et al. 2002; Wolf et al. 2003; Kaulinen 2004; Fugate et al. 2005; Gillis et al. 2006; Moe 2007; Akers y Kaukinen 2009; Instituto Andaluz de la Mujer 2012; Blay 2013; Cala y García 2014; Palermo, Bleck y Peterman 2014; DGVG 2015b; Pontón et al. 2015) muestra que los obstáculos para que las mujeres denuncien la violencia de género que padecen pueden ser tanto internos como externos y tanto de origen social como individual. Entre estas barreras estarían:

- Las dificultades para identificar la violencia padecida. Los patrones de socialización tradicional podrían llevar a algunas mujeres a aceptar o normalizar el maltrato (Hare 2006; Palermo, Bleck y Peterman 2014; DGVG 2015b), haciendo improbable la solicitud de protección (Gracia 2009). Estas dificultades de identificación pueden distorsionar y minimizar la percepción del peligro real que esta violencia puede suponer (Cattaneo 2007; Walker 2012; Cala y García 2014).

- Las estrategias de afrontamiento empleadas para solucionar el problema. La violencia puede generar indefensión y limitar la capacidad de la víctima para actuar o denunciar (Castillejo 2009). Sin embargo, ciertos trabajos (Moe 2007; Blay 2013) cuestionan estos resultados y apuntan que la mayoría de mujeres se enfrentan activamente a la violencia con recursos formales e informales.

- También las creencias erróneas sobre la voluntad de cambio del agresor o su mejoría espontánea, la ausencia de mecanismos de protección, el mal funcionamiento de la justicia, o la inutilidad de denunciar (Blay 2013; Cala y García 2014; DGVG 2015b) dificultarían tomar medidas reales y eficientes para salir de la situación.

- El miedo. En los dos años posteriores a la ruptura de la pareja pueden producirse agresiones, incluso más graves de lo habitual (Walker, Shapiro y Gill 2012). No es extraño que el temor a represalias psicológicas o físicas del maltratador suponga en muchos casos un freno para denunciar (Felson et al. 2002; Harway 2002; Blay 2013; Cala y García 2014; DGVG 2015b).

- El miedo puede referirse también a la posibilidad de que la denuncia fracase y/o a los costos emocionales y personales del proceso posterior (Blay 2013; Cala y García 2014; DGVG 2015b). De hecho, en ocasiones las mujeres acuden al sistema penal para que la violencia cese y la denuncia no siempre lo logra (Hare 2006). Además, frecuentemente desconocen las consecuencias reales de activar este procedimiento, lo que puede llevarlas a desistir o a tomar decisiones contraproducentes (Arenas, Cerezo y Benítez 2013).

- La adherencia emocional. Entre las razones para no denunciar está la complejidad de emociones y sentimientos presentes en la construcción de una relación de pareja (Felson et al. 2002; Díaz y González 2013), que la literatura denomina dependencia emocional y que, en nuestra opinión, se des- 
cribe más adecuadamente como adherencia emocional (Bosch, Ferrer y Alzamora 2006). Su funcionamiento ha sido explicado, entre otros, por el modelo del ciclo de la violencia (Walker 2012).

- La dependencia económica y material. La falta de apoyo y recursos económicos o materiales puede suponer un freno para decidir denunciar (Moe 2007; Lichtenstein y Johnson 2009).

- La tolerancia y el clima social hacia la violencia contra las mujeres en la pareja. La aceptación de la violencia y la culpabilización de las mujeres generan un clima social de tolerancia y legitimación (Policastro y Payne 2013; Gracia 2014; DGVG 2015b). Esto genera una revictimización, aumenta el miedo de las mujeres a no ser creídas por el entorno (DGVG 2015b) y dificulta la denuncia.

- Los sentimientos de vergüenza y culpa. El rechazo social, la discriminación y la estigmatización (Quiles y Morera 2008; Overstreet y Quinn 2013; López 2014) son una de las consecuencias del maltrato. Muchas mujeres se avergüenzan de reconocer públicamente que han tolerado maltrato (especialmente abusos sexuales o humillaciones), que se equivocaron al elegir pareja, $\mathrm{y}$, en general, de ser etiquetadas como maltratadas (Moreira, Boris y Venancio 2011; Blay 2013; Palermo et al. 2014; DGVG 2015b), y esto tiene consecuencias sobre la ocultación o la búsqueda de ayuda (Moreira, Boris y Venancio 2011).

Cabe señalar que las barreras descritas en este epígrafe pueden agravarse y complementarse con otras relacionadas con las circunstancias sociales de las mujeres, como las relacionadas con su etnia, status migratorio y/o socioeconómico, estado de salud, discapacidad y/ o edad (Vives-Cases et al. 2009).

Además, diversos trabajos (v.g. Echeburúa y Corral 2009) señalan que las razones que explican la ausencia de denuncias en casos de violencia de género son las mismas que explican la ausencia de denuncias previas en casos de femicidio íntimo.

En este contexto, el objetivo de este trabajo es contribuir a identificar y comprender las barreras y dificultades con las que se encontraron mujeres víctimas de femicidio íntimo para denunciar la violencia padecida.

\section{Método}

\section{Muestra}

Se identificaron 23 casos de femicidio íntimo ocurridos en Baleares entre 2004 y 2014, incluyendo casos comprobados (con sentencia firme) y otros con indicios suficientes para determinar la identidad del agresor (como confesión explícita). Esto supone una tasa anual en torno a 4,2 mujeres por millón. 
El universo hipotético de estudio estuvo compuesto por todas las personas significativas (familiares y amigos/as) y profesionales (del ámbito comunitario y jurídico) vinculadas a estos casos. Para la realización de este estudio, se realizó un muestreo casual o incidental, es decir, se seleccionaron todas aquellas personas integrantes de ese universo a las que se tuvo acceso.

\section{Variables e instrumento}

Se diseñaron dos modelos de entrevista semiestructurada para aplicarlas, respectivamente, a personas significativas y profesionales. Siguiendo la literatura sobre el tema (Felson et al. 2002; Bonomi et al. 2006; Akers y Kaukinen 2009; Instituto Andaluz de la Mujer 2012; Blay 2013; Cala y García 2014; Sierra et al. 2014; DGVG 2015b), se incluyeron preguntas sobre: características sociodemográficas de víctima y agresor, historia de la relación de pareja, solicitud de ayuda previas, redes de apoyo familiares e institucionales disponibles, y creencias y emociones de las mujeres víctimas de esta violencia.

Sobre la denuncia, a los/as profesionales se les preguntó: «Según su experiencia profesional y su consideración de experto/a, de forma general, ¿Podría indicar alguna de las causas que motivan la escasa tasa de denuncia en el caso de las mujeres asesinadas?» y «¿Podría destacar una de estas causas como principal, generalmente, por encima de todas las demás?». En la entrevista destinada a personas significativas se preguntó, cuando era procedente, «¿Por qué cree que no denunció?».

\section{Procedimiento}

A partir de información de prensa y judicial, se identificaron los 23 casos de femicidio íntimo y un total de 72 personas significativas y profesionales relacionadas con ellos. Se contactó con estas personas, explicándoles los objetivos del estudio, y solicitando su participación. De ellas, 39 (54,17\%) aceptaron participar. La proporción de rechazos fue muy superior entre las personas significativas que entre los/as profesionales. En la Tabla 1 se describen sus características.

Tabla 1

Características de las personas entrevistadas

\begin{tabular}{lccc}
\hline \multicolumn{1}{c}{ Entrevistas } & Hombres & Mujeres & Total \\
\hline Profesionales del ámbito jurídico & 10 & 6 & 16 \\
Profesionales del ámbito comunitario & 4 & 14 & 18 \\
Personas significativas (familiares y /o amigos/as) & 0 & 5 & 5 \\
Total & $\mathbf{1 4}$ & $\mathbf{2 5}$ & $\mathbf{3 9}$ \\
\hline
\end{tabular}


Tras la aceptación, se concertó una cita en el lugar de trabajo de la persona para realizar personalmente las entrevistas, que tuvieron una duración de 30-45 minutos cada una, y fueron grabadas en audio y luego transcritas. Cuando la cita personal no fue posible (en 13 casos), se remitió el cuestionario por correo electrónico para ser respondido.

\section{Análisis de datos}

Todas las entrevistas fueron leídas y codificadas, realizando un análisis cualitativo por categorías a priori (Echeverría 2005), que tomó como referencia las categorías obtenidas en la macroencuesta de 2011 (Ministerio de Sanidad, Servicios Sociales e Igualdad 2012) e incluyó las categorías emergentes surgidas del análisis. A continuación se elaboró un resumen de las categorías obtenidas y del peso otorgado a éstas por las personas entrevistadas, considerando relevantes e incluyendo, por tanto, todas las respuestas de todas ellas (a pesar de que esto podría suponer la sobrerrepresentación de algunos / as participantes).

\section{Resultados}

A continuación se presenta una descripción de las principales características de las mujeres muertas a manos de sus parejas o exparejas en Baleares entre 2004 y 2014 (Tabla 2).

Tabla 2

Características de las mujeres muertas a manos de sus parejas o exparejas en Baleares (2004-2014)

\begin{tabular}{lcc}
\hline & Total & Mujeres que denunciaron \\
\hline \multicolumn{2}{c}{ Características de las mujeres y sus hijos/as } \\
\hline Edad de las mujeres & & \\
20-29 años & $5(21,7 \%)$ & 1 \\
$30-39$ años & $6(26,1 \%)$ & 1 \\
$40-49$ años & $6(26,1 \%)$ & 1 \\
$50-59$ años & $3(13,0 \%)$ & \\
Más de 60 años & $3(13,0 \%)$ & 1 \\
Nacionalidad de las mujeres & & \\
Española & $13(56,5 \%)$ & 2 \\
Extranjera & $10(43,5 \%)$ & 2 \\
Situación laboral de las mujeres & & \\
Activa & $14(60,9 \%)$ & 4 \\
En desempleo & $4(17,4 \%)$ & \\
Jubilada & $2(8,7 \%)$ & \\
No consta & $3(13,0 \%)$ & \\
\hline
\end{tabular}




\section{Total}

Mujeres que denunciaron

\section{Características de las mujeres y sus hijos/as}

Lugar de residencia de las mujeres

Zona urbana - Palma de Mallorca

$\begin{array}{cc}9(39,1 \%) & 2 \\ 14(60,9 \%) & 2\end{array}$

Zona rural

Hijos/as

En común con el agresor

$14(60,9 \%) \quad 3$

De otras relaciones

$7(30,4 \%)$

1

No consta

$2(8,7 \%)$

Edad de los/as hijos/as

Número de menores de 18 años

$24(77,4 \%) \quad 5$

Número de mayores de 18 años

$7(22,6 \%)$

3

Denuncias previas

Sí hay denuncias previas

$4(17,4 \%) \quad 4$

No hay denuncias previas

$17(73,9 \%)$

No consta

$2(8,7 \%)$

\section{Convivencia con el agresor}

$\mathrm{Si}$

No

No consta

$11(47,8 \%)$

$10(43,5 \%) \quad 4$

Tipo de relación con el agresor

Pareja sentimental (noviazgo o matrimonio)

$2(8,7 \%)$

Ex pareja o en trámites de separación

$14(60,9 \%) \quad 2$

Edad de los agresores

18-29 años

$9(39,1 \%) \quad 2$

30-39 años

40-49 años

50-59 años

Más de 60 años

$3(13,0 \%)$

$3(13,0 \%)$

$7(30,4 \%)$

1

$4(17,4 \%)$

$4(17,4 \%)$

No consta

$2(8,7 \%)$

Nacionalidad de los agresores

Española

$14(60,9 \%) \quad 2$

Extranjera

$9(39,1 \%)$

2

Situación laboral de los agresores

Activa

En desempleo

$6(26,1 \%) \quad 2$

Jubilado

$4(17,4 \%)$

$4(17,4 \%)$

No consta

$9(39,1 \%)$

2

Como puede observarse, hay un predominio de mujeres de 30 a 49 años, de nacionalidad española, laboralmente activas, que residían en zona rural, que tenían hijos/as menores en común con su agresor, con quién mantenían una rela- 
ción de pareja y convivían, y cuyo agresor tenía entre 40 y 49 años y una situación laboral indeterminada.

Cabe señalar que sólo 4 de las 23 mujeres asesinadas $(17,4 \%)$ habían interpuesto previamente denuncia por la violencia padecida. Aunque, al igual que ocurre en estudios previos (v.g., Sanz-Barbero et al. 2016), no parece haber características distintivas específicas entre quienes denunciaron y quienes no lo hicieron, en este caso, su número es excesivamente pequeño para obtener conclusiones al respecto.

A continuación se presentan los resultados obtenidos al categorizar las respuestas ofrecidas por las personas profesionales y significativas sobre posibles razones para no denunciar la violencia padecida (Tabla 3).

Tabla 3

Razones para no denunciar en los casos de mujeres muertas a manos de sus parejas o exparejas en Baleares (2004-2014)

\begin{tabular}{lc}
\hline & $\mathbf{n}(\%)$ \\
\hline Dependencia sentimental & $34(\mathbf{1 4 , 4} \%)$ \\
Por amor, porque le quería & $1(0,4 \%)$ \\
Por los hijos / as en común & $7(3,0 \%)$ \\
Por ser el padre de sus hijos & $1(0,4 \%)$ \\
Sentimientos de pena, culpa & $12(5,1 \%)$ \\
Dependencia afectiva & $13(5,5 \%)$ \\
Dependencia económica y material & $\mathbf{1 8 ( 7 , 7 \% )}$ \\
Falta de recursos económicos y medios & $12(5,1 \%)$ \\
Dependencia económica & $6(2,6 \%)$ \\
Miedo al maltratador y a las consecuencias de denunciar & $36(15,3 \%)$ \\
Por miedo al maltratador & $19(8,1 \%)$ \\
Por miedo a las represalias & $8(3,4 \%)$ \\
Incertidumbre sobre qué ocurrirá después de denunciar & $9(3,8 \%)$ \\
Conciencia - concepto de maltrato & $48(20,3 \%)$ \\
Por no tener conciencia de maltrato & $10(4,2 \%)$ \\
No considerarlo maltrato & $5(2,1 \%)$ \\
Por ser maltrato psicológico, no físico & $2(0,8 \%)$ \\
Aceptación $\left(^{*}\right)$ & $5(2,1 \%)$ \\
Justificación al agresor $\left(^{*}\right)$ & $7(3,0 \%)$ \\
Ausencia de percepción del peligro real $\left({ }^{*}\right)$ & $19(8,1 \%)$ \\
Presiones en el ámbito personal y social & $\mathbf{1 7}(7,2 \%)$ \\
Sentimiento de vergüenza & $11(4,7 \%)$ \\
Por presiones o amenazas de la expareja & $1(0,4 \%)$ \\
Por presiones externas, como la familia & $5(2,1 \%)$ \\
Percepción del problema y la solución del maltrato & $\mathbf{6}(\mathbf{2 , 6} \%)$ \\
Creencia que puede arreglarse & $6(2,6 \%)$ \\
Límites culturales, la violencia de género en la sociedad $\left(^{*}\right)$ & $\mathbf{1 5 ( 6 , 4 \% )}$ \\
Valores patriarcales & $2(0,8 \%)$ \\
\hline
\end{tabular}




\begin{tabular}{lc}
\hline & $\mathbf{n}(\%)$ \\
\hline Cultura, educación & $4(1,7 \%)$ \\
Concepción tradicional de la familia & $9(3,8 \%)$ \\
Atención a las víctimas y limitación en los recursos comunitarios y/o jurídicos $\left(^{*}\right)$ & $44(18,6 \%)$ \\
Desconfianza en la justicia & $12(5,1 \%)$ \\
Ineficacia del sistema, revictimización & $8(3,4 \%)$ \\
Poco apoyo institucional & $2(0,8 \%)$ \\
Desconocimiento de los recursos & $9(3,8 \%)$ \\
Falta de medios y recursos preventivos & $6(2,6 \%)$ \\
Falta de formación y sensibilización de los profesionales & $5(2,1 \%)$ \\
Dificultad probatoria del maltrato psicológico & $2(0,8 \%)$ \\
Emociones y pensamientos de la víctima $\left(^{*}\right)$ & $\mathbf{9 ( 3 , 8 \% )}$ \\
Falta de habilidades & $1(0,4 \%)$ \\
Ciclo de violencia & $2(0,9 \%)$ \\
Ambivalencia afectiva & $1(0,4 \%)$ \\
Baja autoestima & $4(1,7 \%)$ \\
Chantaje emocional & $1(0,4 \%)$ \\
Otros & $\mathbf{9 ( 3 , 8 \% )}$ \\
Soledad & $8(3,4 \%)$ \\
Adicciones & $1(0,4 \%)$ \\
\hline
\end{tabular}

(*) Categoría añadida en relación a la macroencuesta de 2011

Las razones a las que se otorga un mayor peso son: en primer lugar, la concepción del maltrato (20,3\%), especialmente, la ausencia de percepción de peligro $(8,1 \%)$; en segundo lugar, las características de los mecanismos para atender a las víctimas y las limitaciones en los recursos de tipo comunitario y/o jurídico $(18,7 \%)$, especialmente, la desconfianza en la justicia $(5,1 \%)$; $y$, en tercer lugar, el miedo al maltratador y a las consecuencia de denunciar $(15,3 \%)$, especialmente, el miedo al maltratador $(8,1 \%)$.

\section{Discusión y conclusiones}

Estos resultados muestran la importante proporción de mujeres que, aun habiendo siendo víctimas de la violencia de género más extrema, no la denunciaron previamente (casi un 85\%), y la coincidencia entre las razones para no hacerlo descritas en la literatura e informadas en este estudio. Así, estos resultados son muy similares a los descritos en la más reciente macroencuesta realizada en España (DGVG 2015a), y en un estudio cualitativo similar realizado en Andalucía sobre 40 mujeres víctimas de violencia de género (DGVG 2015b).

Como ya apuntaban trabajos previos (Felson et al. 2002; Blay 2013; Cala y García 2014; DGVG 2015b), las principales barreras para denunciar la violencia 
padecida serían las creencias erróneas sobre lo que es violencia de género, el modo de hacerle frente y ciertas emociones.

En general, para entender cuáles son estas creencias erróneas y sus posibles efectos, es importante comprender hasta qué punto ser víctima puede afectar al sistema de creencias de la persona, generando cambios (Ruiz-Vargas 2005; Fariña Vilariño y Arce 2014) como: a) Pérdida del sentido de invulnerabilidad, que da paso al miedo, la desesperanza y la ansiedad, y también a una sensación de inseguridad, puesto que, una vez ha ocurrido, es más fácil imaginarse nuevamente en el rol de víctima; b) Modificación de las creencias sobre la propia dignidad, generando una pérdida de la valía personal, y una disminución de la autoestima; y, c) Pérdida de la confianza en los/as demás y, de acuerdo con la hipótesis de la creencia en un mundo justo, aparición de sentimientos de culpa y vergüenza (ValorSegura, Expósito y Moya 2011; Vynckier 2012). Cabe señalar, sin embargo, que ciertos estudios empíricos sobre estos cambios obtienen resultados no concluyentes (Cattaneo et al. 2007; Chapin 2011). Otros trabajos puntualizan que estas creencias básicas sólo quedarían socavadas entre quienes han vivido hechos traumáticos y desarrollado un síndrome de estrés postraumático (Pedersen 2002). Puesto que este síndrome se halla presente en, aproximadamente, la mitad de las mujeres maltratadas, y hasta 3/4 llegan a desarrollar sintomatología (Aguirre et al. 2010; Santandreu y Ferrer 2014), cabe profundizar en el análisis de esta cuestión.

En definitiva, estos resultados nos ayudan a comprender los motivos y razones por los que las mujeres que viven situaciones de violencia de género (incluso en sus formas más extremas que, como en el caso analizado, tienen como resultado su muerte) no la denuncian, y nos permite concluir, como en trabajos previos (Amnistia Internaciona 2012; Cala y García 2014), que las actuaciones básicas para revertir esta situación pasarían, básicamente, por: mejorar la formación de los/as operadores jurídicos/as, garantizando su especialización en violencia de género; y diseñar procedimientos de acompañamiento que garanticen que las mujeres recibirán información y ayuda en todos los pasos de su tránsito por el sistema judicial.

Por otra parte, en el estudio realizado, aparecen también barreras para la denuncia vinculadas a las limitaciones de los recursos comunitarios y / o jurídicos para atender a víctimas de violencia de género, incluyendo: preocupación y malestar de los agentes sociales y jurídicos por la ineficacia del sistema; poco apoyo institucional; ausencia de difusión de los recursos; falta de medios y apoyos preventivos; escasez de comunicación con las víctimas; falta de formación y sensibilización de los/las profesionales; o dificultad para presentar pruebas del maltrato psicológico. Estas barreras generarían en las víctimas desconfianza en la justicia y percepción de poco apoyo institucional por parte de un sistema de justicia «ciego» a la perspectiva de género (Barcons y de las Heras 2015; Castillejo 2009; DGVG 2015b; Díaz y González 2013), dificultando así la denuncia.

Una cuestión importante es que el femicidio íntimo constituye la forma de violencia más extrema contra las mujeres (y sus hijos/as) y tiene también conse- 
cuencias profundas para todo su entorno (OPS 2013). Así, el dolor y la devastación emocional han sido las razones principales por las que gran parte de personas significativas declinó participar en el estudio. Esta dificultad, junto con el reducido tamaño de la muestra en su conjunto, constituyen las limitaciones más relevantes de este estudio, que deberían ser soslayadas con nuevos estudios futuros. Esta constatación ha señalado también la necesidad de ofrecer atención psicológica y psicosocial personalizada y exhaustiva al entorno familiar de las mujeres asesinadas para trabajar tanto el dolor y el daño emocional como otros sentimientos detectados (como la culpa).

Otras limitaciones de este trabajo tienen que ver con: la ausencia de estudios previos en España (Blay 2013) sobre la posible relación entre la reticencia a denunciar la violencia de género y la detectada para otros delitos (Medina 2002); y, dada la constatación (v.g. Palermo, Bleck y Peterman 2014) de diferencias significativas entre países, la necesidad de ampliar este tipo de estudios a contextos geográficos más amplios. Finalmente, sería necesario ampliar el análisis de datos realizado, profundizando en las categorías halladas y en los consensos y disensos en relación a ellas.

\section{Referencias Bibliográficas}

Aguirre, Pamela, Felix Cova, M. Paz Domarchi, Carol Garrido, Ivania Mundaca, Pamela Rincón, Paulina Troncoso, y Paulina Vidal. 2010. «Estrés postraumático en mujeres víctimas de violencia de género». Revista Chilena de Neuropsiquiatría 48(2): 114-122. DOI: https: / / doi.org/10.4067/S0717-92272010000300004

Akers, Caroline, y Catherine Kaukinen. 2009. «The police reporting behavior of intimate partner violence victims». Journal of Family Violence 24(3): 159-171. DOI: https: / / doi.org/10.1007/ s10896-008-9213-4

Amnistía Internacional. 2012. ¿Qué justicia especializada? A siete años de la Ley Integral contra la Violencia de Género: obstáculos al acceso y obtención de justicia y protección. Madrid: Amnistía Internacional. Disponible en https:/ / www.es.amnesty.org/uploads/ media/Que_justicia_especializada.informe_2012.pdf

Arenas, Laura, Ana I. Cerezo, y M. José Benítez. 2013. «Análisis discursivo de los agentes sociales implicados en la violencia de género». Revista Española de Investigación Criminológica 11: 1-28. Disponible en http:// reic.criminologia.net/resumenes.php? numero $=11 \&$ edicion $=2013$

Barcons, María, y Samara de las Heras. 2015. «Justicia sin visión de género igual a IN-justicia. Sobre la insuficiente formación de los operadores jurídicos». Mujeres y Salud 38: 21-23. Disponible en http:/ / mys.matriz.net/mys38/38_sumario.htm

Blay, Ester. 2013. «Voy o no voy: el recurso a la policía en el caso de la violencia de género. Perspectiva de las víctimas». Estudios Penales y Criminológicos 33: 369-400.

Bonomi, Amy E., Victoria L. Holt, Diana P. Martin, y Robert S. Thompson. 2006. «Severity of intimate partner violence and occurrence and frequency of police calls». Journal of Interpersonal Violence 21(10): 1354-1364. DOI: https: / / doi.org/10.1177/0886260506291656

Bosch, Esperanza, Victoria A. Ferrer, y Aina Alzamora. 2006. El laberinto patriarcal. Barcelona: Anthropos. 
Cala, M. Jesús, y María García. 2014. «Las experiencias de las mujeres que sufren violencia en la pareja y su tránsito por el sistema judicial: ¿Qué esperan y qué encuentran?». Anales de la Cátedra Francisco Suárez 48: 81-105. Disponible en http:/ / revistaseug. ugr.es/index.php/acfs/article/view/2781

Casado, Elena. 2012. «Tramas de la violencia de género: sustantivación, metonimias, sinécdoques y preposiciones». Papeles del CEIC 89: 1-28. Disponible en http: / / www.identidadcolectiva.es/pdf/85.pdf

Castillejo, Raquel. 2009. «Intervención legal en violencia de género. En Violencia de género». Tratado psicológico y legal, editado por Francisca Fariña, Ramón Arce, y Gualberto Buela-Casal, 39-59. Madrid: Biblioteca Nueva.

Castillejo, Raquel. 2011. «Problemas que plantea la actual aplicación de la ley integral». En Violencia de género, justicia sustantiva y mediación, editado por Raquel Castillejo, 63124. Madrid: La Ley.

Cattaneo, Lauren B. 2007. "Contributors to assessments of risk in intimate partner violence: How victims and professionals differ».Journal of Community Psychology 35(1): 57-75. DOI: https:/ / doi.org/10.1002/jcop.20134

Cattaneo, Lauren B., Margret E. Bell, Lisa A. Goodman, y Mary Ann Dutton. 2007. «Intimate partner violence victims' accuracy in assessing their risk of re-abuse». Journal of Family Violence 22(6): 429-440. DOI: https:/ / doi.org/10.1007/ s10896-007-9097-8

Chapin, John. 2011. «Optimistic bias about intimate partner violenceamong medical personnel». Family Medicine43(6): 429-432. Disponible en http:/ / www.stfm.org/FamilyMedicine/Vol43Issue6/Chapin429

DGVG - Delegación del Gobierno para la Violencia de Género. 2015a. Macroencuesta de violencia contra la mujer 2015. Avance de resultados. Madrid: Ministerio de Sanidad, Asuntos Sociales e Igualdad. Disponible en http://www.violenciagenero.msssi. gob.es/violenciaEnCifras/macroencuesta2015/home.htm

DGVG. 2015b. Sobre la inhibición a denunciar de las víctimas de violencia de género. Madrid: Ministerio de Sanidad, Asuntos Sociales e Igualdad. Disponible en http: / / www.violenciagenero.msssi.gob.es/laDelegacionInforma/pdfs/DGVG_informa_Inhibicion_Denunciar_Victimas_VG_pdf

Díaz, Capitolina, y Rosario González. 2013. «Agresores, agresiones y agredidas: la violencia de género». En Análisis interdisciplinar de la violencia de género, editado por Yolanda Fontanil, M. Angeles Alcedo, y Janine Roberts, 11-18. Oviedo: KRK.

Echeburúa, Enrique, y Paz Corral. 2009. «El homicidio en la relación de pareja: análsis psicológico». Eguzkilore 23, 139-150. Disponible en: http: / / www.ehu.eus / documents / 1736829/2176697/ 12-Echeburua.indd.pdf [consultado em 19.09.2016].

Echeverría, Genoveva. 2005. Apuntes Docentes de Metodología de Investigación: Análisis cualitativo por categorías. Santiago de Chile: Universidad Academia de Humanismo Cristiano. Disponible en: https://www.academia.edu/9444115/Apuntes_Docentes_de_ METODOLOGIA_DE_INVESTIGACION_ANALISIS_CUALITATIVO_POR_CATEGORIAS [consultado el 19 de septiembre de 2016].

EIGE - European Institute for Gender Equality. 2014. Administrative data sources on genderbased violence against women in the EU Current status and potential for the collection of comparable data. Vilnius: EIGE.

Fariña, Francisca, Manuel Vilariño, y Ramón Arce. 2014. «Evaluación psicológica forense de la victimización». En Justicia terapéutica: experiencias y aplicaciones, editado por David B. Wexler, Francisca Fariña, Luz A. Morales, y Sara P. Colín, 161-174. Puebla: Asociación Iberoamericana de Justicia Terapéutica.

Felson, Richard B., Steven F. Mesner, Anthony W. Hoskin, y Glenn Deane. 2002. «Reasons 
for reporting and not reporting domestic violence to the police». Criminology 40(3): 617-648. DOI: https: / / doi.org/10.1111/j.1745-9125.2002.tb00968.x

Ferrer, Victoria A., Esperanza Bosch, y Teresa Riera. 2006. «Las dificultades en la cuantificación de la violencia contra las mujeres en la pareja: Análisis psicosocial». Revista de Intervención Psicosocial y Calidad de Vida 15(2): 181-201. Disponible en http: / / scielo.isciii.es/pdf/inter/v15n2/v15n2a05.pdf

FRA - European Union Agency for Fundamental Rights. 2014. Violence against women: an EU-wide Survey. Main results. Luxembourg: Publications Office of the European Union. Disponible en http:/ / fra.europa.eu/en/publication/2014/violence-againstwomen-eu-wide-survey-main-results-report

Fugate, Michelle, Leslie Landis, Kim Riordan, Sara Naureckas, y Barbara Engel. 2005. «Barriers to domestics violence help seeking. Implications for intervention». Violence Against Women 11(3): 290-310. DOI: https: / / doi.org/10.1177/1077801204271959

García-Moreno, Claudia. 2005. Estudio multi-país de la OMS sobre salud de la mujer y violencia doméstica. Primeros resultados sobre prevalencia, eventos relativos a la salud y respuestas de las mujeres a dicha violencia. Ginebra: OMS. Disponible en http: / / www.who.int/gender/violence/who_multicountry_study/summary_report / summaryreportSpanishlow.pdf

García-Moreno, Claudia, y Henrica Jansen. 2009. Challenges in measuring violence against women. Pre-Conference Workshop 3, SVRI Forum 2009, Johannesburg, South Africa, 6 July. Disponible en http:/ / www.svri.org/forums / forum2009/presentations / workshop3.pdf

García-Moreno, Claudia, Christina Pallitto, Karen Devries, Heidi Stöckl, Charlotte Watts, y Naheema Abrahams. 2013. Global and regional estimates of violence against women: prevalence and health effects of intimate partner violence and non-partner sexual violence. Geneva: World Health Organization. Disponible en http:/ / www.who.int/iris/handle/10665/ 85239

Gillis, Joseph R., Shaindl L. Diamond, Paul Jebely, Victoria Orekhovksy, Ellis M. Ostovich, Kristin MacIsaac, Sandra Sagarti, y Deborah Mandell. 2006. «Systemic obstacles to battered women's participation in the judicial system. When will the status quo change?». Violence Against Women 12(12): 1150-1168. DOI: https:// doi.org/10.1177/ 1077801206293500

Gracia, Enrique. 2009. Violencia doméstica contra la mujer: el entorno social como parte del problema y de su solución. En Violencia de género. Tratado psicológico y legal, editado por Francisca Fariña, Ramón Arce y Gualberto Buela-Casal, 75-85. Madrid: Biblioteca Nueva.

Gracia, Enrique. 2014. «Intimate partner violence against women and victim-blaming attitudes among Europeans». Bulletin of the World Health Organization 92: 380-381. DOI: https:/ / doi.org/10.2471/BLT.13.131391

Hare, Sara C. 2006. «What do battered women want? Victim's opinions on prosecution». Violence \& Victims 21(5): 661-624.

Harway, Michele. 2002. Intimate Partner Abuse and Relationship Violence. Washington: American Psychological Association. Disponible en: http://www.apa.org/about/ division/activities / partner-abuse.pdf [consultado el 14 de marzo de 2016].

Instituto Andaluz de la Mujer. 2012. La renuncia a continuar en el procedimiento judicial en mujeres víctimas de violencia de género: Un estudio en la Comunidad Autónoma Andaluza. Sevilla: Instituto Andaluz de la Mujer. Disponible en https://www.juntadeandalucia.es/institutodelamujer/ugen/node/2133

Laliga, Mónica, y Amparo Bonilla. 2015. «Políticas públicas en el tratamiento de la violen- 
cia de género: una aproximación crítica a la eficacia de las herramientas jurídicas y alternativas». Journal of Feminist, Gender and Women Studies 1: 41-51. Disponible en http: / / revistas.uam.es/ revIUEM/article/view / 410

Kaulinen, Catherine. 2004. The help-seeking strategies of female violent crime victims: The direct and conditional effects of race and the victim-offender relationship. Journal of Interpersonal Violence 19(9): 967-990. DOI: https: / / doi.org/10.1177/0886260504268000

Lichtenstein, Bronwen, e Ida M. Johnson. 2009. «Older African American women and barriers to reporting domestic violence to law enforcement in the rural deep South». Women and Criminal Justice 19(4): 286-305. DOI: https: / / doi.org/10.1080 / 08974450903224329

López, Alma. 2014. Violencia de género: posible revisión a partir de la noción de estigma femenino. En Justicia restaurativa y violencia de género: más allá de la Ley Orgánica 1/2004, editado por Raquel Castillejo, M. Jesús Sande, y Cristina Torrado, 41-48. Madrid: La Ley.

Manjoo, Rashida. 2012. Report of the Special Rapporteur on violence against women, its causes and consequences. A/HRC/20/16. United Nations. General Assembly. Disponible en http:/ / www.ohchr.org/Documents/Issues/Women/A.HRC.20.16_En.pdf [consultado el 14 de marzo de 2016].

Medina, Juan José. 2002. Violencia contra la mujer en la pareja: investigación comparada y situación en España. Valencia: Tirant Lo Blanc.

Meil, Gerardo. 2012. Análisis sobre la macroencuesta de violencia de género 2011. Madrid: Ministerio de Sanidad, Servicios Sociales e Igualdad. Disponible en http: / / www.violenciagenero.msssi.gob.es/violenciaEnCifras / estudios/investigaciones/2011/ estudio/macroencuesta2011.htm

Ministerio de Sanidad, Servicios Sociales e Igualdad. 2012. Macroencuesta sobre violencia de género 2011. Principales resultados.

Moe, Angela M. 2007. «Silenced voices and structured survival: battered women's help seeking». Violence Against Women 13(7): 676-699. DOI: https://doi.org/10.1177/ 1077801207302041

Naredo, María. 2009. «¿Son realidad los derechos de las mujeres ante la violencia de género?». Crítica 960 (Marzo-Abril): 27-31. Disponible en http://www.revista-critica.com/archivo

Novo, Mercedes, y Dolores Seijo. 2009. «Aproximación psicosocial a la violencia de género: aspectos introductorios». En Violencia de género. Tratado psicológico y legal, editado por Francisca Fariña, Ramón Arce y Gualberto Buela-Casal, 63-74. Madrid: Biblioteca Nueva.

ONU - Organización de las Naciones Unidas. 2012. Vienna Declaration on Femicide. Vienna Symposium on Femicide. 26 November. Disponible en: http:/ / www.genevadeclaration.org / fileadmin/ docs / Co-publications / Femicide_A\%20Gobal\%20Issue \% 20that\%20demands\%20Action.pdf [consultado el 14 de marzo de 2016].

OPS - Organización Panamericana de la Salud. 2013. Femicidio.. Washington: Organización Mundial de la Salud. Disponible en http: / / apps.who.int/iris/bitstream / 10665/98828/1/WHO_RHR_12.38_spa.pdf

Overstreet, Nicele M., y Diane M. Quinn. 2013. «The Intimate Partner Violence Stigmatization Model and Barriers to Help-Seeking». Basic Applied Social Psychiatry 35(1): 109122. DOI: https:/ / doi.org/10.1080/01973533.2012.746599

Palermo, Tia, Jennifer Bleck, y Amber Peterman. 2014. «Tip of the Iceberg: Reporting and Gender-Based Violence in Developing Countries». American Journal of Epidemiology 179(5): 602-612. DOI: https: / / doi.org/10.1093/aje/ kwt295

Pedersen, Duncan M. 2002. «Political violence, ethic conflict, and contemporary wars: 
broad implications for health and well-being». Social Science and Medicine 55(2): 175190. DOI: https: / / doi.org/10.1016/S0277-9536(01)00261-1

Policastro, Christine, y Brian K. Payne. 2013. «The Blameworthy Victim: Domestic Violence Myths and the Criminalization of Victimhood». Journal of Aggression, Maltreatment \& Trauma 22(4): 329-347. DOI: https: / / doi.org/10.1080/10926771.2013.775985

Pontón, Paloma, Inma Pastor, Ana Acosta, y Ángel Belzunegui. 2015. «Femicide and gender based violence in Spain». En Finding solutions for a post crisis society, editado por Teresa Torres, Ángel Belzunegui, y Josep Moreno, 308-325. Tarragona: Universitat Rovira i Virgili.

Quiles, M. Nieves y M. Dolores Morera. 2008. «El estigma social: la diferencia que nos hace inferiores». En Método, teoría e investigación en psicología social, editado por José Francisco Morales, Carmen Huici, Ángel Gómez, y Elena Gaviria, 377-399. Madrid: Pearson Educación.

Ruiz-Vargas, José M. 2005. Recuerdos traumáticos: el enemigo interior. En Madrid 11-M. Un análisis del mal y sus consecuencias, editado por Amalio Blanco, Rafael del Aguila, y José Manuel Sabucedo, 311-352. Madrid: Trotta.

Russell, Diane. 2013. "Femicide" - The Power of a Name. En Femicide. A global issue that demands action, editado por Academic Council of the United Nations System (ACUNS), 19-20. Viena: ACUNS. Disponible en http:// www.genevadeclaration. org / fileadmin / docs / Co-publications / Femicide_A\%20Gobal\%20Issue\%20that $\% 20$ demands\%20Action.pdf

Santandreu, Marta, y Victoria A. Ferrer. 2014. «Effectiveness of cognitive behavioral treatment for posttraumatic stress disorder on women victims of intimate partner violence». Behavioral Psychology 22(2): 239-256.

Sanz-Barbero, Belén, Julio Heras-Mosteriorc, Laura Otero-García, y Carmen Vives-Cases. 2016. «Perfil sociodemográfico del feminicidio en España y su relación con las denuncias por violencia de pareja». Gaceta Sanitaria 30(4): 272-278. DOI: https:/ / doi.org/10.1016/j.gaceta.2016.03.004

Sierra, Juan Carlos, M. Paz Bermúdez, Gualberto Buela-Casal, José M. Salinas, y Fredy S. Monge. 2014. «Variables asociadas a la experiencia de abuso en la pareja y su denuncia en una muestra de mujeres». Universitas Psychologica 13(1): 37-46. DOI: https: / / doi.org/10.11144/Javeriana.UPSY13-1.vaea

Spinelli, Barbara. 2013. Femicide in Europe. En Femicide. A global issue that demands action, editado por Academic Council of the United Nations System (ACUNS), 42-46. Viena: ACUNS. Disponible en http: / / www.genevadeclaration.org/fileadmin/ docs / Co-publications / Femicide_A\%20Gobal $\%$ 20Issue $\% 20$ that $\% 20$ demands $\%$ 20Action.pdf

Valor-Segura, Inmaculada, Francisca Expoósito, y Miguel Moya. 2011. «Victim blaming and exoneration of the perpetrator in domestic violence: The role of beliefs in a just world and ambivalent sexism». The Spanish Journal of Psychology 14(1): 195-206. DOI: https:/ / doi.org/10.5209/rev_SJOP.2011.v14.n1.17

Vives-Cases, Carmen, Diana Gil-González, Juncal Plazaola-Castaño, María Isabel Montero-Piñar, Isabel Ruiz-Pérez, Vicenta Escribà-Agüir, Gaby Ortiz-Barreda, Jordi Torrubiano-Domínguez. 2009. «Violencia de género en mujeres inmigrantes y españolas: magnitud, respuestas ante el problema y políticas existentes». Gaceta Sanitaria 23(1),100-106. DOI: https: / / doi.org/10.1016/j.gaceta.2009.07.008

Vynckier, Gerwynde. 2012. Victim Blaming Revisited: Beyond the Explanation of Self-Protection. /International Perspectives in Victimology 7(1): 38-46. Disponible en http: / hdl.handle.net/1854/LU-3072307 
Walker, Leonore E. A. 2012. «Descripciones de la violencia y el ciclo de la violencia». En El sindrome de la mujer maltratada, editado por Leonore E. A. Walker, 145-170. Bilbao: Descleé de Brouwer.

Walker, Leonore E. A., David Shapiro, y Kelley Gill. 2012. «Evaluación de riesgos y potencia letal». En El sindrome de la mujer maltratada, editado por Leonore E. A. Walker, 171-220. Bilbao: Descleé de Brouwer.

Wolf, Marsha E., Uyen Ly, Margaret A. Hobart, y Mary A. Kernic. 2003. «Barriers to seeking police help for intimate partner violence». Journal of Family Violence 18(2): 121-129 DOI: https: / / doi.org/10.1023/ A:1022893231951

Ferrer Pérez. Doctora en Psicología y Catedrática de Psicología Social de Género de la Universidad de las Islas Baleares (UIB). Coordinadora y profesora del Máster Universitario en Políticas de Igualdad y Prevención de la Violencia de Género de la UIB y coordinadora para esta universidad del Doctorado Interuniversitario en Estudios Interdisciplinares de Género. Miembro del grupo de investigación «Estudios de género» de la UIB.

Esperanza Bosch Fiol. Doctora en Psicología y Profesora Titular de Psicología Básica de la Universidad de las Islas Baleares (UIB). Directora del Máster Universitario en Políticas de Igualdad y Prevención de la Violencia de Género de la UIB. Directora de la Oficina para la Igualdad de Oportunidades de la UIB. Directora de la Cátedra para el Estudios de la Violencia de Género de la UIB. Investigadora principal del grupo de investigación «Estudios de género» de la UIB.

Artículo recibido el 14 de marzo de 2016 y aceptado para publicación el 15 de septiembre de 2016. 